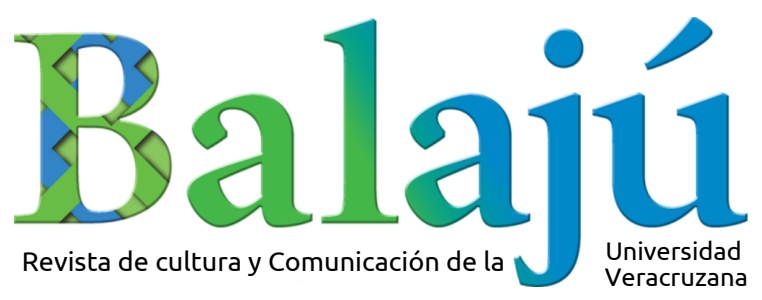

\title{
Radio Teocelo como esfera pública alternativa: apropiación tecnológica y movilizaciones sociales
}

\section{José Agustín Castellanos Rodríguez}

Universidad Iberoamericana

Recibido: 07-08-2020 | Aprobado: 30-11-2020 


\title{
Balajú
}

\section{Radio Teocelo como esfera pública alternativa: apropiación tecnológica y movilizaciones sociales}

\section{Radio Teocelo as an Alternative Public Sphere: Technological Appropriation and Social Mobilizations}

José Agustín Castellanos Rodríguez ${ }^{1}$

\section{Resumen}

En un esfuerzo por generar un análisis sustancial sobre los procesos de movilización social, los estudios parecen haber obviado el amplio ecosistema mediático a través del cual las comunidades han visibilizado sus identidades y objetivos, y coordinado acciones colectivas hacia intereses compartidos, de forma autónoma respecto del Estado. En este artículo, se analizan las capacidades de los actores sociales para apropiar las tecnologías de comunicación y reconfigurarlas como esferas públicas alternativas desde las cuales mediar los contenidos mediáticos hegemónicos y hacer contrapeso al control absoluto del Estado y de la industria privada.

Palabras clave: Radio comunitaria, nuevos movimientos sociales, esfera pública, ecología de medios

\begin{abstract}
In their efforts to analyze particular processes of social mobilization, researchers seem to have overlooked the broad media ecosystem through which communities have made their identities and objectives visible and coordinated collective actions towards shared interests, independent of State intervention. This article analyzes the capacities of social actors to appropriate communications technologies and reconfigure them as alternative public spheres from which to mediate hegemonic media content and counterbalance the absolute control of the State and private industry.
\end{abstract}

Keywords: Community radio, new social movements, public sphere, media ecology

\footnotetext{
${ }^{1}$ Universidad Iberoamericana. ORCID: 0000-0001-6123-1150, agosto0420@gmail.com
} 


\title{
Balajú
}

\section{RAdio TeOcelo COMO ESFERA PÚBlica Alternativa: APROPIACIÓN TECNOLÓGICA Y MOVILIZACIONES SOCIALES}

\author{
José Agustín Castellanos Rodríguez
}

\section{Introducción}

Desde el supuesto de la radio comunitaria como plataforma de acción y espacio de organización en procesos de movilización hacia fines e intereses compartidos, en la trama cotidiana (Atton, 2002; Downing, 2001), son de relevancia las capacidades sociales que apropian la tecnología radiofónica para el despliegue de acciones colectivas. Se sugiere que la radio comunitaria puede constituir una esfera pública para la visibilización de los intereses de las comunidades que han sido excluidas de los procesos de deliberación política y de representación en los espacios públicos y privados. Sin embargo, la categoría de radio comunitaria no pretende generar una conceptualización absoluta sino, por el contrario, exponer las características y experiencias particulares, derivadas de tácticas de uso y de apropiación en un amplio ecosistema mediático, en contextos y situaciones específicas.

Se asume que la producción y el consumo de contenidos han servido como un mecanismo de integración, con base en la reproducción de significados compartidos por un colectivo (Martín-Barbero, 1987). Es necesario problematizar el concepto de comunidad como un territorio simbólico - no solo físico-donde se comparten actividades, intereses y problemas afianzados en la vida cotidiana, lo cual reafirma los sentidos de identidad, correspondencia y solidaridad (de Certeau, 2000; Violich y Astica, 1971).

En ese proceso, la diferencia de identidades culturales entre comunidades se hace evidente (de Certeau, 2000). De acuerdo con García Canclini (2006), el consumo se interpreta como el lugar donde los grupos compiten por la apropiación del producto social, acentuado por la desigual participación en la estructura de producción. En un sistema social, las comunidades "excluidas" y "no excluidas" disputan la capacidad de visibilizar sus intereses políticos y sociales en el espacio público, dentro de una estructura de orden social. El conflicto se extiende hacia el sistema de producción de contenidos, los cuales están cargados de sentidos, de acuerdo con el colectivo que los produce (Martín-Barbero, 1987). 


\section{Balajú}

A través del caso de Radio Teocelo, se interroga la capacidad de la radio comunitaria para representar una esfera pública desde la cual generar productos que visibilicen los intereses comunes y organicen movilizaciones hacia objetivos compartidos.

El análisis pretende delimitar el concepto de comunidad e integrar una perspectiva que reconozca las distinciones derivadas de las formas de apropiación y uso de productos hegemónicos, dentro de un sistema de integración social sustentado en un marco de sentidos y generado por la compartición de significados, inserto a su vez en un sistema de mayor amplitud donde se compite por la representación social, política y cultural (Martín-Barbero, 1987; de Certeau, 2000; García Canclini, 2006). Se propone, además, documentar cómo, frente a la supuesta incapacidad y desinterés de los medios comerciales y públicos por satisfacer las necesidades de las comunidades (Peppino, 1990), estas han apropiado estrategias tecnológicas como instrumentos de comunicación que faciliten la movilización social y la visibilización de demandas comunes. Desde este paradigma, se reconoce la capacidad interactiva del amplio ecosistema de medios, con principal atención en la radio comunitaria como espacio que facilita la comunicación y la organización dentro de las movilizaciones sociales.

\section{Aproximaciones teóricas: comunidad}

El análisis integral de una práctica comunicativa que depende de una noción cuya conceptualización goza de una amplitud y ambigüedad tan basta como su abundancia semántica en los diversos contextos en los que se ha aplicado (Downing, 2000) exige una demarcación particular que, si bien no pretende generar una abstracción normativa, busca sellar los horizontes de su interpretación sin comprometer el campo de sus posibilidades. Es necesaria una revisión que aporte coordenadas teóricas ante la polifonía de discursos y la diversidad de posibilidades tanto estructurales como psicosociales comprendidas bajo el concepto de comunidad (Almeida y Sánchez, 2014).

El acercamiento a "comunidad" ha enfrentado controversias grabadas por las diversas aplicaciones y disciplinas científicas desde las que se han generado aproximaciones teóricas y prácticas, hasta el punto de comprometer su vigencia, utilidad y capacidad operativa, no obstante conceptualizaciones basadas en dicotomías configuradas por constructos políticos, económicos y sociales derivados de la modernidad, que acentuaban antagonismos entre lo rural y lo urbano, lo orgánico y lo mecánico, lo tradicional y lo moderno y lo afectivo y lo racional (Almeida y Sánchez, 2014; Parra, 2017). 


\section{Balajú}

Desde las ciencias sociales se ha conceptualizado la comunidad como una diversidad de formas de organización y convivencia relacionadas con su ubicación espacial y temporal (Almeida y Sánchez, 2014); un lugar donde se desarrolla la vida y se generan interacciones que intervienen la misma. Cada disciplina ha incluido u obviado aspectos, en mayor o en menor medida, relevantes para su objeto de estudio: ejes estructurales, relaciones psicosociales, agrupamientos humanos, espacios geográficos, significados y hábitos (Causse, 2009).

Aun cuando la transdisciplinariedad es su distintivo, y así el concepto de comunidad es construido y deconstruido, sus componentes objetivos y subjetivos, producidos por el cruce del eje estructural (espacio, tiempo, materialidad y relaciones de poder) y psicosocial (intersubjetividad, inconsciente y formas de reconocimiento/diferenciación), comparten una crítica a la crisis evidente de la modernidad. Y, a pesar de que la reminiscencia romántica de la comunidad se aventura, los fundamentos de la vida común, reflejo de formas colectivas de pensar, sentir, actuar y aceptar interacciones, conflictos y utopías, son una respuesta alternativa al desarrollo neoliberal. Así, se reconoce una idea compuesta por elementos estructurales y psicosociales que afectan a sus miembros, un espacio para la reconstitución del lazo social, sustrato del comunitarismo frente a la manifestación individualista de la sociedad. En concreto, la comunidad es el concepto desde el cual se representa un espacio cotidiano de autonomía y de resistencia (Almeida y Sánchez, 2014; Duarte, 2017; Parra, 2017).

Por otro lado, el concepto de comunidad se ha desprendido de delimitaciones geográficas, sin comprometer el sentido de identidad, una identidad intersubjetiva como unidad de pensamiento y emoción, producida en función de intereses, símbolos y códigos comunes, que ha generado experiencias inéditas de comunitarismo mediante lazos de solidaridad a través de geografías diferenciadas. Dicha demarcación permite identificar campos de acción basados en la construcción o apropiación de espacios para el desarrollo de prácticas comunitarias. En dichos campos, el "espacio" no está determinado por una localidad; sin embargo, las prácticas ahí desarrolladas pueden intervenir los lugares que sí son espacios físicos (Almeida y Sánchez, 2014; Martín-Barbero, 1987).

Aunque Martín-Barbero (1987) y de Certeau $(1999,2000)$ no refirieron a la comunidad sino al barrio como espacio rodeado por la modernidad dominante, sus análisis sobre las prácticas culturales cotidianas pueden extenderse hacia la comprensión de la identidad comunitaria. En ese espacio, Martín-Barbero (1987) distinguió los procesos de reconocimiento y diferenciación para la constitución de identidades, un lugar estructurado por formas 


\section{Balajú}

específicas de sociabilidad e interacción, donde se establecen elementos básicos para la construcción de un nosotros, acentuado en intensos momentos de movilización (Melucci, 1999).

De tal forma, a pesar de que el territorio ha sido el eje central para muchas aproximaciones teóricas y operativas al concepto de comunidad, delimitado como el lugar donde se desarrolla la vida social, resultan de interés los procesos de identificación y de diferenciación que permiten procesar experiencias y aspiraciones individuales y colectivas, a partir de las cuales se genera el sentido mismo de comunidad: percepción de identidad y pertenencia a una estructura simbólica de relaciones sociales, desde la cual es posible activar la solidaridad y el apoyo mutuo hacia objetivos compartidos (Almeida y Sánchez, 2014; Parra, 2017; Causse, 2009; Socarrás, 2004).

Tras un breve análisis del concepto, se recalca la tesis de Masip (2011) al referir que el origen del sentido socioconceptual de la comunidad se ha perdido por causa de su uso indiscriminado para referir una multiplicidad de fenómenos, y así ha sido rebasado. En cambio, su condición performativa se mantiene vigente en lo propio de su sentido. Es decir, al referir la comunidad, se afirma la existencia de algo cuya comprensión involucra elementos que facilitan su entendimiento común. Si bien la polisemia del concepto puede referir a un sistema de relaciones psicosociales, un agrupamiento humano o un espacio geográfico específico, su interpretación se puede demarcar por el énfasis depositado en sus elementos estructurales o subjetivos. De acuerdo con el carácter subjetivo, se trasciende la determinación de una localización geográfica específica y se prioriza el sentido de identidad, nutrido por la interacción y la organización de sus miembros en la recreación de significados en la vida cotidiana, vinculados con procesos sociales y políticos hacia objetivos compartidos (Causse, 2009).

Por lo tanto, se refiere a la comunidad no como un concepto, sino como un proceso sociocultural, heterogéneo y dinámico, desarrollado por una colectividad no siempre inserta en un marco territorial, con estructuras horizontales que facilitan las relaciones de cooperación y coordinación, con el fin de desarrollar acciones colectivas, formales e informales, enfocadas en intereses, objetivos y problemas comunes (Korsbaek, 2009; Melucci, 1999; Socarrás, 2004). 


\section{Balajú}

\section{Movimientos sociales, apropiación y mediaciones}

A partir de la definición propuesta, se problematizan las condiciones y parámetros -normativos o espontáneos- de identidad colectiva que negocian el despliegue de tácticas de acción y la conformación de movimientos sociales (Melucci, 1999). No obstante, en primera instancia, se destaca la importancia de delimitar el concepto de "apropiación" (de Certeau, 1999; 2000).

La apropiación ha sido referida por de Certeau (2000) como una producción independiente de sentido. Un proceso artesanal de construcción de frases propias, desarrollado por los usuarios a partir de vocabularios y sintaxis recibidas de la economía cultural dominante: innumerables formas de transformar la autoridad de acuerdo con intereses propios. Estos procedimientos populares juegan con los dispositivos de disciplina y modifican su funcionamiento mediante tácticas basadas en lo cotidiano, a través de las cuales apropiar el espacio de producción.

Al centrar la atención en la dinámica de producción, apropiación y uso, Martín-Barbero (1987) distinguió un espacio donde ocurren movimientos de mediación: asimilación, rechazo, negociación y refuncionalización de productos que exigen la cooperación con el lector para ser significados, de acuerdo con parámetros del ámbito cotidiano. Esa serie de movimientos es intervenida por el desarrollo de la vida, en escenarios decodificadores microsociales, como la comunidad. Es ahí donde se reproduce el sentido de identidad, mediante pactos de lectura sostenidos por una trama de redes de comunicación e interacción que tejen relaciones de correspondencia y de solidaridad. No obstante, la mediación implica la reconfiguración de tecnologías de producción y la resignificación de los contenidos (Lievrouw, 2011). Al apropiar un dispositivo, los sujetos reconfiguran el proceso de producción para sus propios fines, mientras que, a través de la mediación, crean nuevos sentidos a los productos hacia significados comunes (Lievrouw, 2011; Mattoni y Treré, 2014).

El concepto de mediación comprende el consumo como un espacio de convivencia y conflicto, donde el uso de los bienes encarna formas de transmisión de sentidos, evidencia de la disputa entre grupos a propósito de la desigual participación en la estructura productiva y por la apropiación del producto social y sus formas de uso (Martín-Barbero, 1987; García Canclini, 2006). Los procesos de mediación permiten restaurar la capacidad de agenciación de los actores sociales, responder a los mecanismos de exclusión y reivindicar aquellos colectivos marginados de la estructura dominante. En este caso, las comunidades pueden romper el modelo lineal de producción-consumo y constituir espacios alternativos de colaboración y de 


\section{Balajú}

representación frente a un orden hegemónico. Este tipo de asociaciones no se agota en las fronteras espaciales y, en muchos casos, articula la percepción y la solución de problemas hacia proyectos sociales de mayor amplitud (Martín-Barbero, 1987; Mattoni y Treré, 2014).

Desde las formas de consumo, uso y apropiación, sustentadas en la mediación de sentidos, se genera una relación descriptiva hacia la activación de sistemas de acción, desarrollados en función de una identidad. La identidad colectiva, como proceso, incorpora operaciones de producción y apropiación de estructuras cognoscitivas para la identificación y el cálculo de costos/beneficios relativos a los fines, medios y ámbitos de las acciones que una comunidad puede desarrollar; de activación de relaciones de cooperación y de solidaridad entre actores, quienes interactúan, se comunican, negocian y adoptan decisiones; y la realización de inversiones emocionales que permitan el reconocimiento bajo un mismo sentido, a través de prácticas cotidianas (Melucci, 1999; Milan, 2008). Dichas operaciones, al incluir inversiones emocionales, no se reducen a un cálculo material; en su cotidianidad radican las "formas de hacer", artesanales, tradicionales y propias, las cuales reproducen repertorios de acciones colectivas, sustentadas en los modos de ejecutar prácticas no discursivas: maneras de comunicar, habitar y movilizarse (de Certeau, 2000; Melucci, 1999).

Por consiguiente, la acción colectiva se interpreta como una inversión asociativa derivada de procesos de identificación de los límites y potencialidades de una comunidad, los cuales dan sentido a las expectativas compartidas, una acción conjunta en defensa de intereses

e identidades comunes que parte de la voluntad de un sujeto, cuya intención individual coincide con el interés colectivo y, por lo tanto, cuya participación persigue objetivos generales (Melucci, 1999; Revilla, 1996). Las acciones colectivas, estructuradas en sistemas de operación, activan movimientos sociales en respuesta a tensiones que disturban el equilibrio estructural (Melucci, 1999).

Conforme a la definición de Arato y Cohen (2001) de sociedad civil como una comunidad determinada por procesos culturales particulares, que actúa en el espacio público hacia intereses compartidos, con operaciones y objetivos independientes al Estado, la composición de movimientos sociales constituye el proceso mediante el que dichas comunidades buscan realizar sus objetivos. Los movimientos sociales no son objetos concretos; al contrario, son procesos en continua evolución en los que los actores despliegan acciones constantes de acuerdo con diferentes temporalidades y objetivos (Mattoni y Treré, 2014; Melucci, 1999). 


\section{Balajú}

Bajo la noción de Nuevos Movimientos Sociales (NMS) se reconocen un conjunto de acciones colectivas que cuestionan la comprensión materialista de las perspectivas utilitaristas marxistas, en particular los esquemas de los movimientos obreros y de movilización de recursos (Arato y Cohen, 2001; Berrío, 2006; Melucci, 1999). El concepto inscribe acciones no institucionales, desarrolladas por individuos que comparten creencias, identidades o prácticas cotidianas, mediante redes de comunicación, interacción y solidaridad fuera del control estatal, caracterizadas por la cooperación autónoma hacia intereses posmateriales, ajenos a la clase, la incorporación institucional y la racionalidad instrumental (Lievrouw, 2011; Melucci, 1999). Los NMS poseen un carácter derivado de marcos de significado, pautas de relación social, formas de organización comunitaria (Laraña, 1999; Melucci, 1999) y "lógicas de acción basadas en la política, la ideología y la cultura, y otras fuentes de identidad como la etnicidad, el género o la sexualidad" (Berrío, 2006: 229).

Asimismo, los NMS acentúan el conflicto entre grupos y formas de comunicación, por lo que tienden a orientarse hacia la apropiación de espacios públicos, medios de producción y dispositivos mediáticos, con el fin de generar áreas de movimiento desde donde visibilizar identidades y objetivos (Arato y Cohen, 2001; Diani, 1992; Melucci, 1999). Nutridos por una inteligencia anterior, los actores extienden prácticas sociales con capacidad de modificar y reutilizar los contenidos preexistentes en el espacio tecnocrático, y crear condiciones favorables mediante operaciones emancipadoras (de Certeau, 2000; Mattoni y Treré, 2014), acciones transgresoras, adscritas al universo de la capacidad inventiva y el uso de objetos con cierto grado de autorreflexión, que trascienden los límites de la planificación instrumental que las organizaciones funcionalistas imponen para reducir la heterogeneidad (Cassigoli, 2016; de Certeau, 2000).

En concordancia, Lievrouw (2011) detalla el uso de tecnologías de comunicación en procesos de movilización social, como espacios de organización, comunicación y visibilización de objetivos. El análisis de los NMS exige contemplar las tecnologías de comunicación reconfiguradas dentro del repertorio de acciones colectivas, como espacios alternativos desde donde generar y expresar opiniones, coordinar acciones y materializar ideales políticos (Lievrouw, 2011; Mattoni y Treré, 2014).

En general, la aproximación al concepto de mediación posibilita abarcar una variedad de prácticas mediáticas, ejecutadas por actores sociales en espacios alternativos, producidos por la apropiación de los rígidos sistemas funcionales hacia objetivos comunes (Lievrouw, 


\section{Balajú}

2011; Mattoni y Treré, 2014). La atención se centra en la reconfiguración de los medios de comunicación apropiados y en las formas de producción y mediación de contenidos. Milan (2013) utiliza el concepto de "prácticas emancipadoras de comunicación" como acciones colectivas que generan alternativas a las infraestructuras de comunicación existentes. Así, bajo la denominación de medios alternativos (Lievrouw, 2011) o tecnologías liberadas (Milan, 2013), se refieren instrumentos de comunicación que han sido reconfigurados, a partir de prácticas emancipadoras, para desafiar los formatos hegemónicos y la forma en que abordan los asuntos políticos, económicos, culturales y sociales. Desde la teoría de los NMS se reconoce que los medios de comunicación no representan simples instrumentos o medios hacia un fin, sino espacios para la deliberación y la acción social, y plataformas para la relación y el conflicto con otras “esferas públicas” (Mattoni, 2017; Mattoni y Treré, 2014; Lievrouw, 2011).

\section{Radio comunitaria}

El papel de los medios, como tecnologías apropiadas por las comunidades, evidencia las distinciones derivadas del sistema de producción y, por lo tanto, las acciones colectivas de reconfiguración para la institución de espacios alternativos a la infraestructura hegemónica (Lievrouw; 2011; Mattoni y Treré; 2016; Milan, 2013). A través de prácticas mediáticas, los actores sociales pueden interactuar con los medios de comunicación con el fin de desplegar acciones de reconocimiento y de visibilización, y aumentar su capacidad de organización en una amplia gama de situaciones y contextos (Mattoni, 2017; Mattoni y Treré, 2014; Milan, 2008).

A pesar de que la literatura respecto a prácticas mediáticas en los movimientos sociales puede incorporar diversas formas de relación a través de un amplio ecosistema de medios, canales, contenidos y prácticas a través de los cuales los actores interactúan en entornos de movilización y despliegan transacciones simbólicas (Mattoni, 2017; Mattoni y Treré, 2014 y 2016; Treré, 2012), aquí la atención se centra en la apropiación y la reconfiguración de la tecnología radiofónica por comunidades marginadas de los espacios de representación pública.

La radio comunitaria ha sido referida como una "tecnología liberada", resultado de "prácticas emancipadoras de comunicación" (Milan, 2013): una apropiación sustentada en redes de comunicación, interacción y solidaridad para la deliberación autónoma ante los medios hegemónicos y el control estatal; una plataforma de representación de identidades, intereses y objetivos comunes (Mattoni, 2017). Sin embargo, definir la radio comunitaria representa la 


\section{Balajú}

incorporación de una serie de atributos ubicados en una estrecha veta que, con facilidad, puede generar falacias y alimentar una idealización indulgente (Downing, 2001). En una reducción esencialista, los medios comunitarios no son intrínsecamente buenos; deben ser alentados a definir sus objetivos y propósitos en el marco de su ejercicio cotidiano (Milan, 2009). Si bien la noción de comunidad es el rasgo distintivo que define dicha forma de comunicación, esta es configurada por la presencia, en menor o en mayor grado, por distintas propiedades sociales, políticas, económicas y geográficas, exclusivas de cada experiencia.

Es oportuno incluir a la radio comunitaria en un heterogéneo ecosistema mediático que incorpore diversas prácticas de comunicación, además de operaciones de acción directa y subversión de dispositivos mediáticos, que pueda dar cuenta de formas de comunicación desde la interpersonal y la impresa hasta formatos híbridos electrónicos, en un modelo que privilegie el potencial transformador de los medios y que evidencie las relaciones entre múltiples tecnologías, actores y prácticas comunicativas (Mattoni, 2017; Milan, 2013; Treré, 2012; Treré y Mattoni, 2016), prácticas asentadas sobre sistemas previos de relación cotidiana (conversaciones, exhibiciones, manifestaciones) que han evolucionado a través de múltiples dispositivos y sistemas de significación (carteles, panfletos, mensajes de audio) que interactúan entre sí (Scolari, 2012), flujos multidireccionales de comunicación en un diverso repertorio de acciones y de medios apropiados (Mattoni y Treré, 2016).

Desde una perspectiva ecologista, se pueden interpretar los medios como especies que interactúan en un mismo ecosistema mediático, en relación con actores y sus prácticas comunicativas; esto permite expandir el enfoque unitario que ha centrado su atención en la apropiación de una sola tecnología de comunicación, una abstracción agudizada en los recientes estudios sobre movimientos sociales y plataformas digitales. El rol de los medios debe ser analizado dentro de procesos comunicativos más amplios y en relación con otras prácticas mediáticas acrónicas y sincrónicas (Treré, 2012). Por otra parte, esta perspectiva también permite observar cómo las tecnologías, prácticas y redes de comunicación han evolucionado hacia nuevos sistemas de significación que interactúan entre sí (Mattoni, 2017; Mattoni y Treré, 2014; 2016; Treré, 2012).

Pese a su diversidad, los dispositivos mediáticos han sido referidos bajo diversos conceptos, de acuerdo con las aproximaciones teóricas que los han abordado, en relación con múltiples contextos: medios alternativos (Atton, 2002), radicales (Downing, 2000), ciudadanos (Rodríguez, 2011) o comunitarios (Calleja y Solís, 2005; Gumucio, 2004). En todo caso, las 


\section{Balajú}

denominaciones parten del reconocimiento de una alternativa al estándar hegemónico (Atton, 2002). Y, aunque Downing (2000) ha priorizado la denominación radical para enfatizar las capacidades de cambio social de los medios y de sus "activistas", mientras que Rodríguez (2011) ha sumado la denominación ciudadana para resaltar la identificación política, estos espacios oposicionales de participación, a pesar de distanciarse en algunos elementos, mantienen objetivos compartidos y dirigidos hacia la identidad colectiva, la participación social y la representación propia, enfocados en la búsqueda de transformaciones sociales en múltiples dimensiones (Atton, 2002; Downing, 2000; Milan, 2008).

Ahora bien, se prioriza la noción comunitaria al aludir a prácticas de la vida cotidiana, procesos de interacción, comunicación e identidad colectiva, centrados en definiciones compartidas, las cuales determinan la orientación y el campo de las acciones sociales (Downing, 2000; Milan, 2008). A pesar de ubicarse en contextos específicos, la construcción de comunidad no se limita a un entorno geográfico, sino a un espacio social de reproducción y representación de sentidos e intereses comunes para la creación y el refuerzo de lazos sociales para el cambio (Milan, 2008; 2009).

Las posibilidades conquistadas a través de la apropiación de la radio y su reconfiguración como una herramienta política incluyen una serie de procesos de empoderamiento, mediante los cuales se potencializa la participación de las comunidades a través de las tecnologías liberadas y la mediación de contenidos hacia intereses comunes (Lievrouw, 2011; Milan, 2013). La radio comunitaria es un espacio de representación, al que pueden recurrir múltiples comunidades y movimientos sociales, en diversas situaciones y contextos (Mattoni, 2017; Milan, 2008).

El empoderamiento expone un proceso en el que, a través de prácticas emancipadoras, los actores toman control, apropian diversas tecnologías mediáticas y reconfiguran la comunicación. El factor de poder aparece ligado a la capacidad de las comunidades para incrementar sus habilidades de expresión, participación, deliberación y organización sobre su espacio social, para conquistar objetivos comunes (Lievrouw, 2011; Milan, 2013).

Por lo tanto, como la Asociación Mundial de Radios Comunitarias-México (AMARCMX) (2008) ha declarado, la comprensión de las radios comunitarias depende de su identificación como espacios de "relaciones políticas"; al buscar la transformación social, desarrollan un proyecto que cuenta con objetivos, metodologías y estrategias, "en el sentido de que asume compromisos y toma posición respecto a la problemática concreta en que vive" 


\section{Balajú}

(Calleja y Solís, 2005: 24). Pese a que las radios comunitarias puedan compartir el objetivo general de transformar la realidad social (Milan, 2008), las diferencias y características existentes, entre las formas concretas de accionar la tecnología, están determinadas por el contexto de cada experiencia: "Algunas están vinculadas con los intereses de los sectores que sufren la desigualdad económica y viven en la pobreza. Otras ponen el acento en las desigualdades producto de las diferencias de género" (Lamas, 2003: 5).

Un análisis de la apropiación de los medios de comunicación, desde los NMS, permite trascender una perspectiva instrumentalista y llegar a una interpretación como esferas públicas de acción y deliberación, para la representación de identidades, objetivos y puntos de vista alternativos o radicales (Lievrouw, 2011; Milan, 2013).

Pese a la variable particularidad de identidades y caracterizaciones de cada radio comunitaria, se reconoce que lo que define a una emisora como comunitaria es su capacidad de extender operaciones cotidianas para la constitución de espacios públicos, autónomos respecto de las determinaciones del Estado y del mercado, para la deliberación y el consenso como acción política (Milan, 2008; 2013); la reafirmación de la identidad colectiva, mediante significados comunes dentro de un proceso participativo (Lievrouw, 2011); el desarrollo de acciones colectivas en función de objetivos y problemáticas compartidas (Downing, 2000; Guo, 2015); y el ejercicio de las capacidades sociales para la modificación de las condiciones contextuales (Calleja y Solís, 2005; Lamas, 2003; López, 1995).

\section{Radio comunitaria como esfera pública}

La comprensión de la radio comunitaria como una esfera pública autónoma, y a veces en conflicto con los espacios regidos por las lógicas del Estado y del mercado (Gasparello, 2011; Krohling, 2012; MhagaMa, 2015; Milan, 2013), se fundamenta en una serie de críticas al sentido de lo "público" en las democracias deliberativas desarrolladas en el capitalismo tardío, y a la constitución de espacios institucionales, orientados por la teoría de Jürgen Habermas. Las críticas cuestionan la distinción absoluta público-privado, un sistema que depende de exclusiones de acceso, respecto a diferencias de estatus en una escala social de participación, y la concepción unitaria de una esfera central, que obvia la existencia de múltiples espacios de representación social (Downing; 2000; Fraser, 1999; Guo, 2015).

Los cuestionamientos evidencian que la constitución de lo público se establece en una distinción antagónica frente a la vida privada, con fundamentos sustanciales en la ciudad- 


\section{Balajú}

Estado griega, social y políticamente consolidada. Desde sus orígenes conceptuales, en la teoría de Habermas (1981; 1998), la representación de lo público se configuró por circunstancias temporales y estructurales delimitadas, protagonizadas por una sociedad burguesa (Downing, 2000; Rabotnikof, 2011): "industrialmente avanzada y social-estatalmente constituida; un 'tipo ideal', homogéneo" (Habermas, 1981: 41).

A pesar de la idealización recurrente que dio sentido a lo público, del supuesto de Habermas $(1981 ; 1998)$ se desatacan, como punto de partida, los conceptos fundamentales que configuran la esfera pública. Basado en la poli griega como forma orgánica, Habermas (1981) la concibe como un espacio para la congregación del pueblo y la conformación de la opinión de representación (Sermeño, 2013). Dicha esfera implica una red abierta de opiniones formuladas en lenguaje natural, donde la sociedad civil conforma la opinión pública; a partir de esos criterios, se destacan la accesibilidad general y el interés y la utilidad común. Por lo tanto, la esfera o espacio público simboliza una red metalocal con dimensiones sociales para el encuentro popular, mediante la comunicación y la deliberación democrática, con el fin de elaborar opiniones y juicios colectivos respecto a problemáticas comunes (Habermas, 1981; 1998; Sermeño, 2013).

Para Habermas $(1981 ; 1998)$, la deliberación democrática representa una herramienta de inclusión y de participación, determinada por reglas y procedimientos comunes. Un proceso comunicativo orientado a la conformación de la opinión pública, basada en el diálogo y en el consenso, para su representación y amplificación. La deliberación depende de la participación en el intercambio racional de opiniones. Por lo tanto, Habermas (1981) asume la opinión pública como el producto de la voluntad y el derecho de la sociedad civil a manifestar opiniones propias, de forma racional y argumental, respecto a asuntos de interés general. Por su parte, Arato y Cohen (2001) han distinguido la sociedad civil como una trama asociativa, libre de interferencia estatal y económica, sustentada en la deliberación hacia objetivos comunes.

A pesar de lo normativo del concepto, Fraser (1999) parte del principio de esfera pública habermasiana como una propuesta crítica que ofrece herramientas para el análisis de los movimientos sociales y las teorías políticas asociadas a ellos. No obstante, recalca que la distinción categórica entre público-privado ha excluido ciertos asuntos de la vida cotidiana de ser deliberados como legítimos del interés público. Sumado a esto, cuestionó que dicha esfera dependió de contextos occidentales altamente industrializados, "sincrónica aplicación a las circunstancias de la sociedad burguesa" (Habermas, 1981: 41). Pese a la retórica acerca de la 


\section{Balajú}

accesibilidad del supuesto habermasiano, la esfera pública se ha configurado por una serie de exclusiones significativas; las condiciones regulativas de la esfera institucional no han sido ecuánimes: aspectos como clase, género, raza y etnicidad son criterios de exclusión del espacio de deliberación democrática (Fraser, 1999).

En general, las condiciones de la esfera pública ilustrada, en la burguesía occidental, no corresponden con la diversidad de las sociedades actuales. Habermas idealizó su concepción mientras ignoró otros ámbitos que coexistieron con el burgués (Downing, 2000; Fraser, 1999). Por lo tanto, "los intereses y las opiniones individuales se han ido organizando en grupos que demandan representación como [...] portavoces políticos y mediáticos” (Sampedro, 2000: 38), con el fin de generar arenas discursivas para amplificar identidades, intereses, necesidades e interpretaciones antagónicas, a través de contradiscursos (Atton, 2002).

En oposición a la unitaria esfera pública, bajo la noción de "contra-públicos subalternos", Fraser (1999) planteó la existencia de múltiples "contra-sociedades civiles": asociaciones voluntarias evidencia de la existencia de diversas esferas alternativas, en competencia con el espacio burgués e institucional. Dichos espacios son derivados de la disparidad de acceso y de participación en la deliberación entre grupos desiguales, puesto que la esfera habermasiana está configurada por mecanismos de exclusión y por relaciones estructurales de dominación y subordinación, basadas en diferencias de estatus social y capital cultural (Atton, 2002; Downing, 2000).

Como dimensiones adicionales, los espacios alternativos entablan relación con la esfera institucional sin significar la participación dentro del poder, sino una actividad paralela, autónoma, de vigilancia y crítica sobre quienes lo detentan. En principio, esa naturaleza extragubernamental fue lo que confirió la sensación de autonomía y legitimidad a la opinión pública de la esfera burguesa. Sin embargo, su construcción única y esencialista limita las acciones sociales de intervención en la vida política. Las esferas sostenidas en la participación colectiva pueden contrarrestar las formas de exclusión de la esfera central, constituida por instituciones estatales. Así, las contra-esferas, de libre acceso y participación se fundamentan en identificar a la sociedad civil como el ámbito de organización y solidaridad que reafirma la identidad comunal y expresa posicionamientos respecto a asuntos de interés común, de forma autónoma respecto del Estado y del mercado (Fraser, 1999; Sampedro, 2000).

A la conceptualización de la opinión pública, Grossi (2007) ha sumado que esta no se agota en la interacción entre referentes sociales, sino que incorpora un amplio ecosistema 


\section{Balajú}

mediático en el cual se generan interacciones y se despliegan transacciones simbólicas a través de redes desde donde dirigir esfuerzos sociales (Scolari, 2012). Es decir, los elementos sistémicos de las dinámicas de producción de opinión no se agotan en su dimensión deliberativa; necesitan de soportes comunicativos, canales "en los cuales la opinión pública se forma y se difunde, se exhibe y se invoca públicamente" (Grossi, 2007: 109).

Reconocer el protagonismo de los medios ubicados en los terrenos del orden dominante (de Certeau, 2000), los cuales han excluido a la sociedad civil y se han convertido en estructuras a favor de los poderes homogeneizadores, acentúa la necesidad de apropiación de tecnologías para la participación, deliberación y producción independiente de sentidos, de forma autónoma ante el Estado y el mercado (Nightingale, 2011; Sampedro, 2000). La opinión pública, expresada a través de canales de comunicación, es útil para la acción colectiva al ser más estable, duradera y menos susceptible a perturbaciones de los medios dominantes y de la agenda política (Koopmans, 2004).

Así, organizaciones de la sociedad civil, como los NMS, generan y median mensajes para visibilizar su identidad y sus objetivos (Lievrouw, 2011). De esta forma, el despliegue de acciones colectivas puede derivar en la constitución de esferas públicas materializadas en sistemas de comunicación, sobre soportes comunitarios de identidad (Sampedro, 2000). No obstante, es fundamental identificar la evolución de redes de comunicación interpersonal hacia sistemas de medios. La apropiación y la operación de los medios se establece sobre redes cotidianas de interacción y de comunicación, con anterioridad existentes en el mundo de la vida (Atton, 2002; Downing, 2000).

Mientras que las críticas expuestas no fueron desarrolladas desde la comprensión de la radio comunitaria, se reconoce su capacidad para amplificar la opinión pública, derivada de la deliberación, con orientaciones alternas a las prácticas mediáticas institucionales y privadas (Milan, 2008; 2013). A través de la radio comunitaria, como esfera alterna, es posible describir la realidad social y visibilizar necesidades e identidades comunes, en un ejercicio de participación colectiva. En la medida en que contra-públicos subalternos emerjan, en respuesta a las exclusiones de las esferas dominantes, se expande el espacio discursivo en las sociedades estratificadas. La existencia de espacios subalternos no representa posicionamientos en total independencia de las esferas hegemónicas, sino plataformas autónomas en constante diálogo y conflicto (Fraser, 1999). 


\section{Balajú}

Al priorizar la noción comunitaria, se aluden procesos de interacción, comunicación e identidad colectiva respecto a sentidos compartidos y campos de acción social, independientes de las determinaciones del Estado y del mercado (Downing, 2000; Milan, 2008). De esta forma, la apropiación de la radio y su reconfiguración como una plataforma de acción política incorpora procesos cotidianos de participación, deliberación y representación de identidades, objetivos y necesidades comunes (Lievrouw, 2011). La radio comunitaria es un espacio abierto de conformación y representación de la opinión pública, sostenido en la colaboración de la sociedad civil, al que pueden acceder múltiples comunidades y movimientos sociales en diversas situaciones y contextos, para dialogar con esferas institucionales e influir en procesos sociales de mayor amplitud, sin condicionar su autonomía (Arato y Cohen, 2001; Mattoni, 2017; Milan, 2008). A través de estas contra-esferas, de libre acceso e intervención, se reafirma la identidad comunal, se representan posicionamientos respecto a asuntos de interés común y se visibilizan identidades excluidas de la esfera central (Fraser, 1999).

En definitiva, en tanto la operación cotidiana de un medio, apropiado y reconfigurado por la acción colectiva, esté enfocado en la reafirmación de la identidad y en el ejercicio de las capacidades sociales, en función de intereses y problemáticas compartidas, y su acceso y participación no estén determinados por mecanismos de exclusión (Fraser, 1999), se recalca su comprensión como una esfera pública alternativa: un espacio autónomo para la deliberación, como acción política y social, y una plataforma de visibilización y de relación con otras esferas. A través de la radio comunitaria como esfera pública alternativa, es posible desafiar los poderes dominantes de los campos hegemónicos, en espacios donde la colaboración y las expresiones de identidad son ejes para compartir el conocimiento y rechazar los regímenes de control estatal y mercantil (Lievrouw, 2011; Mattoni, 2017; Mattoni y Treré, 2014; Milan, 2013).

No obstante, la constitución de una esfera pública a partir de la operación de una emisora comunitaria depende de representar un espacio - no físico- sustentado sobre redes cotidianas de interacción y de comunicación, en donde asuntos privados, manifiestos en la vida cotidiana, en función de sentidos, intereses, identidades, objetivos, problemas y necesidades comunes sean dialogados y deliberados como acción política y social, para la conformación de consenso. Dicha trama asociativa debe sustentarse en principios de accesibilidad y de participación que no estén condicionados por mecanismos de exclusión ni por relaciones de dominación y de subordinación. 


\section{Balajú}

\section{Teocelo y su radio}

A partir de las aproximaciones teóricas, se presentó un análisis sobre los medios de comunicación como espacios apropiados por la acción colectiva de comunidades, movilizadas hacia objetivos e intereses compartidos. La intención de este apartado es evidenciar el potencial de los supuestos teóricos, a través del caso particular de la emisora comunitaria Radio Teocelo.

La revisión bibliográfica se contrastó con una etnografía compuesta por seis meses de observación participante, fruto de la experiencia vivencial del autor, y dos entrevistas semiestructuradas aplicadas a Élfego Riveros Hernández en distintos tiempos (1 de septiembre de 2017 y 13 de mayo de 2020), con el objetivo de evidenciar contrastes y coincidencias. En la primera entrevista, Riveros se desempeñaba como director de Radio Teocelo y presidente de la Asociación Veracruzana de Comunicadores Populares (AVERCOP), concesionaria de la emisora; en la segunda, como consejero de la AVERCOP y miembro de la emisora. Su experiencia es de relevancia, al atestiguar el desarrollo de la vida cotidiana en relación con procesos de apropiación social, con principal interés en Radio Teocelo.

La entrevistas semiestructuradas se desarrollaron a partir de una guía de interés, dentro de la cual el investigador tuvo la libertad de profundizar en aspectos relevantes que permitieran introducir preguntas adicionales para la obtención de información, sin condicionar la libertad expresiva del entrevistado ni su facultad descriptiva respecto de acontecimientos y actividades que pudieran haber escapado a la observación participante (Fontana y Frey, 2012; Hernández, Fernández y Baptista, 2014).

Las entrevistas fueron transcritas y analizadas, a partir de la codificación abierta, para después contrastarse con los referentes producidos de la revisión literaria y de la observación, con el fin de generar una perspectiva más amplia del fenómeno estudiado. La codificación abierta facilita la descomposición y el análisis minucioso de datos, con el fin de identificar y conceptualizar los significados contenidos en el texto, en términos de similitudes y de contrastes (Seid, 2016). La interpretación de datos puede diferir de otras investigaciones o percepciones, debido a la particularidad del fenómeno, la subjetividad de los individuos y las condiciones del contexto analizado, aspectos que brindan una naturaleza cualitativa a la investigación.

Cabe recalcar constantes perspectivas internas y externas, con el fin de minimizar la influencia que pudiera ejercer el contexto. Debido a la previa colaboración en la emisora y en la comunidad de Teocelo, Veracruz, se omitió una inmersión inicial en el ambiente; se ha 


\section{Balajú}

constatado el desarrollo de procesos sociales y se ha generado un punto de vista "interno" (Hernández et al., 2014). Por lo tanto, se recalca el interés al abordar el fenómeno desde una perspectiva cualitativa y se destaca que los objetivos están orientados hacia la descripción, la explicación y la comprensión de fenómenos en contextos determinados.

Radio Teocelo, ubicada en la cabecera del municipio de Teocelo, en la zona centro montañosa del estado de Veracruz, es la primera emisora comunitaria constituida en México y la primera en obtener un permiso de tipo cultural para uso del espectro radioeléctrico, de acuerdo con el antiguo sistema de permisos y concesiones de la Ley Federal de Radio y Televisión.

El análisis de la emisora se presenta a través de un orden cronológico que sustente cómo el surgimiento de Radio Teocelo respondió a circunstancias políticas y sociales de la transición de la década de los cincuenta a los sesenta: un contexto nacional marcado por movilizaciones obreras, campesinas y estudiantiles (Hernández, 2013; Olmedo, 2016; Ramos, 2007). La emisora fue el resultado de la necesidad de reforzar la identidad comunitaria en un ambiente de rezago ante el cacicazgo y la insuficiencia institucional (Sosa, 2011).

En el periodo de constitución de la emisora, los contenidos radiofónicos y la operación de las infraestructuras eran determinados por un reducido grupo centralizado de empresarios, encabezados por Emilio Azcárraga Vidaurreta, con el sustento de una fracción político social referida como "burguesía política mexicana" (Mejía, 1989). De esta forma, el funcionamiento de las estaciones radiofónicas, en todo el país, era marcado por las presiones derivadas del vínculo de sujeción entre los industriales de la radio y el Estado mexicano, a través del establecimiento y la modificación discrecional de pautas de legislación (Mejía, 1989; Romo, 1991).

El control empresarial sobre el ejercicio radiofónico favoreció el desinterés institucional por dotar al Estado de un instrumento de comunicación social propio, lo que reforzó el control absoluto de la radio en manos de un grupo centralizado de empresarios y perpetuó su potestad sobre los canales de producción y de difusión de contenidos (Mejía, 1989). Bajo dicho panorama, Riveros relata que la identidad y los intereses de la localidad de Teocelo, una zona de pobreza acentuada por la crisis de la actividad cafetalera - de la cual depende la economía de la región-, no fueron incluidos ni representados por el ejercicio radiofónico centralizado. 


\section{Balajú}

Frente a dicha invisibilización, a inicios de la década de los cincuenta, un colectivo de habitantes del municipio de Teocelo fundó el Grupo Promotor de Asistencia Social, con la intención de promover la identidad colectiva a través de proyectos comunitarios autónomos respecto de la administración municipal: una caja de ahorro popular, una biblioteca pública, la edición de la Revista Teocelo y una cooperativa de vinos de naranja (Altavoz, 2015). En años posteriores, el mismo colectivo constituyó el Centro de Promoción Social y Cultural (CEPROSOC), bajo el cual comenzó la operación de la XEYT, radio cultural campesina, ahora Radio Teocelo (Arias-Godínez, 1990).

De acuerdo con Riveros, el surgimiento de la emisora derivó de la acción colectiva, en respuesta al desinterés institucional por atender las necesidades comunitarias. Una acción de contrapeso al poder: pese a que el Estado tiene sus propias formas de expresión, la comunidad tuvo la urgencia de generar espacios desde donde exhibir el contraste que se hacía evidente en la realidad cotidiana. De forma simultánea, a través de la radio, el periódico Altavoz y Teocelo Te Ve, como recursos dentro del ecosistema de significación, la comunidad generó espacios de interacción y de expresión para la cultura, la identidad, la defensa del territorio y el interés común. Aunque algunos soportes tecnológicos se hayan transformado, incluso extinto, las prácticas de comunicación se han mantenido constantes y se han trasladado a otros dispositivos.

En 1989, se conformó la AVERCOP, y la XEYT se renombró Radio Teocelo (Altavoz, 2015). Riveros distingue que la conformación de la AVERCOP destacó tres derechos particulares para la comunidad: el derecho a comunicar, a través de un ecosistema mediático, en cuyos medios la comunidad pudo orientar sus pensamientos, expresiones e intereses. Radio Teocelo, el periódico Altavoz y Teocelo Te Ve, como repertorio de medios, evidenciaron las relaciones simultáneas entre tecnologías, actores y prácticas comunicativas extendidas a partir de redes previas de relación y de comunicación, para el despliegue de esfuerzos sociales dentro de un mismo sistema de significación. Estas apropiaciones fueron producto de la evolución de formas anteriores de comunicación e interacción sobre redes de identidad, correspondencia y solidaridad, afianzadas en la vida cotidiana y reforzadas a través de varios medios que conviven de forma simultánea y que generan diversas formas de relación (Scolari, 2009). Por otro lado, fueron producto del derecho a la libertad de expresión, ejercido a plenitud, con responsabilidad y con ética, pero sin censura ni limitación alguna, y del derecho a la información, es decir, que lo que no informan los medios comerciales o públicos lo informa la radio comunitaria, al 


\section{Balajú}

colocar en el ámbito público aquello que es de interés para la comunidad y que permite a los ciudadanos incidir en su vida política.

La emisora, desde sus inicios, ha pensado en ser útil a la comunidad y en servir como un espacio público de colaboración, para reforzar la identidad colectiva mediante la publicidad de expresiones culturales y políticas, afirma Riveros. Mientras que los medios privados buscan la rentabilidad económica y los públicos la rentabilidad política, los medios comunitarios apuestan por la rentabilidad social, cultural y política, con el fin de que las comunidades la apropien dentro de su repertorio de herramientas en su vida cotidiana.

El sentido de comunidad, en Radio Teocelo corresponde a un lazo de identidad y de solidaridad en zonas con pocos recursos, con economías depreciadas, agobiadas por el desempleo y la migración. Oyentes, locutores, productores y reporteros conforman una comunidad guiada por intereses comunes, en un proceso en constante mediación. En este caso, la comunidad no se reduce al municipio de Teocelo; a pesar de no contar con un sofisticado sistema de medición de audiencias, a través de la participación y el contacto, se valora que la emisora es un potencial medio de información para 400 localidades rurales de 10 municipios, con un aproximado a los 500 mil habitantes, detalla Riveros.

Al respecto, Calleja y Solís (2005) han recalcado que Radio Teocelo es una tecnología que ha sido apropiada para satisfacer las necesidades y problemas comunes de múltiples localidades; "El objetivo de Radio Teocelo es promover el desarrollo integral de las comunidades, por lo que la emisora crea vínculos entre ciudadanos, grupos de la sociedad civil, instituciones públicas, privadas, parroquias y ayuntamientos que busquen el mismo fin" (Calleja y Solís, 2005: 216).

De acuerdo con Olmedo (2016), un claro ejemplo de su función social como espacio público de debate es el programa Cabildo Abierto que, desde 1997, brinda la oportunidad para el ejercicio de la transparencia y la rendición de cuentas:

Una vez a la semana, los ayuntamientos que se encuentran en el área de cobertura de la emisora acuden a informar sobre las obras que se están llevando a cabo, el ejercicio del presupuesto y las acciones de gobierno en torno a conflictos sobre el agua, tenencia de la tierra y programas de apoyo. Con teléfono al aire, la población puede preguntar, cuestionar y aportar sobre el desempeño de las autoridades locales y sus programas; al mismo tiempo las autoridades tienen un canal abierto para informar con detalle sus acciones, a través de un medio que les permite 


\section{Balajú}

llegar a todos, especialmente a las comunidades de más difícil acceso y que, a final de cuentas, son las directamente involucradas (Calleja y Solís, 2005: 25).

Resultado de la constancia de Cabildo Abierto, en mayo de 2004 Radio Teocelo fue acreedora del Premio Nacional de Periodismo, en la categoría de Orientación y Servicio a la Sociedad. De igual forma, la Secretaría de la Función Pública (SFP) reconoció el trabajo de la radio por ser un proyecto operado por la sociedad civil, que impulsa la no corrupción y la rendición de cuentas (Hernández, 2013). En ese mismo año, el Centro Mexicano para la Filantropía A. C. (CEMEFI) otorgó el reconocimiento Mira por los demás, por el servicio brindado por y para la comunidad (Calleja y Solís, 2005). En 2005, la Organización de las Naciones Unidas para la Educación, la Ciencia y la Cultura (UNESCO, por sus siglas en inglés) reconoció a Radio Teocelo como un espacio público que, a través de contenidos locales, incide en las políticas públicas de la región (Calleja y Solís, 2005).

A pesar de que las referencias pasadas podrían aludir a un nexo institucional, la operación de Radio Teocelo es posible gracias al subsidio y a la participación comunitaria (Hernández, 2013). La emisora depende del trabajo colaborativo, mediante redes de solidaridad y a través de donaciones de sus oyentes, dentro de las limitaciones de financiamiento establecidas en los siete apartados del artículo 89 de la Ley Federal de Telecomunicaciones y Radiodifusión. Riveros destaca el hecho de reconocer que una radio comunitaria es un medio autónomo; no intervienen ni las empresas ni los gobiernos ni ningún otro tipo de interés más allá del comunitario. Las comunidades que la han apropiado, en diversos contextos y situaciones, confían en ella, la apoyan, la financian y la defienden cuando se mete en problemas, "y vaya que ha habido problemas por posturas asumidas ante gobiernos municipales, estatales o federales", agrega Riveros.

Un aspecto que posibilita la constante reconfiguración de la tecnología radiofónica es la mediación de significados. Los contenidos de Radio Teocelo son determinados y constituidos por la misma comunidad, en función de los sentidos, intereses, identidades, objetivos, problemas y necesidades comunes, evidentes en la vida cotidiana. La emisora fomenta la acción colectiva a través de un proceso colaborativo de difusión de información, manifestaciones culturales y capacitaciones, con el fin de permitir a los individuos y a las comunidades una constante apropiación y resignificación del medio (Olmedo, 2016). 


\section{Balajú}

Así, diversos actores sociales y comunidades han representado sus reivindicaciones a través de la tecnología radiofónica y han coordinado acciones colectivas hacia objetivos determinados, producto de la mediación de aspectos culturales, informativos e identitarios. La función de Radio Teocelo no se limita a la difusión de información local, su acción se encuentra en la reivindicación de aspectos culturales, políticos y sociales, donde intervienen los radioescuchas (Olmedo, 2016):

... como un elemento que potencia la amplitud de movimientos sociales y el impacto de las acciones colectivas realizadas. Por ejemplo, Radio Teocelo ha tenido un papel importante respecto a la movilización de los habitantes en contra de los intentos de privatización de recursos naturales (agua) de la zona; no hablamos solo se difundir el hecho, sino que ayuda en la cohesión del movimiento más allá del auge emocional de los líderes. Es decir, se pasa de una multitud a un grupo organizado que actúa ya no por espontaneidad, sino de manera constante para alcanzar un objetivo en común (Olmedo, 2016: 1286).

Con dichos fines, el diseño de programación como un modelo dinámico se estructura mediante la participación. Aunque la emisora no cuenta con metodologías sofisticadas para delimitar un público meta y un rating, los contenidos están enfocados en los sentidos que establecen una identidad colectiva. Además de orientar, informar y entretener, deben ayudar a quitar vendas de los ojos, para que pasen cosas en bien de la comunidad, de las familias y de la región, una construcción obligada a atender las necesidades e intereses de los radioescuchas ya sea por cartas, correos electrónicos, visitas, sondeos, foros- pero, en principio, por el acercamiento, la participación y las redes de vinculación de las comunidades con la emisora.

Un aspecto que distingue la labor de los radialistas comunitarios es su uso de fuentes de información, el cual visibiliza perspectivas sociales alternas a las oficiales, aquellas que no suelen ser representadas en los medios institucionales y privados. Desde la apropiación de la radio por la acción colectiva, como una plataforma de visibilización, hay una mediación que establece los términos y condiciones de la comunicación en los espacios y recursos que esta controla (Baca-Feldman, 2014). A lo largo de sus 16 horas de transmisión diaria, los miembros de Radio Teocelo visibilizan los problemas y necesidades comunes a través de:

... noticias locales, transmisiones a control remoto de fiestas patronales, debates políticos, programas con una perspectiva de género, barras infantiles, espacios de 


\section{Balajú}

jóvenes, protestas ciudadanas, entrevistas con alcaldes, encuentros deportivos, promoción de la salud, defensa del medio ambiente, cooperativismo, combate a la corrupción y promoción de la democracia en todas sus formas (Calleja y Solís, 2005: 216).

De igual forma, las capacidades de Radio Teocelo se han extendido y han incidido en el espacio político, dentro del campo de posibilidades y límites de las acciones comunitarias. El ejercicio de Radio Teocelo se ha definido en la medida de su construcción como un espacio de encuentro, autónomo frente a la intervención estatal; una plataforma de representación de objetivos compartidos y de construcción de consenso (Calleja y Solís, 2005). Diversas formas de organización, movilización y acción no institucionales se ha apropiado la emisora para generar interlocución entre múltiples comunidades. Algunas acciones colectivas han sido las movilizaciones de los habitantes de la región en contra de los intentos de privatización de los recursos naturales (Olmedo, 2016); la coordinación en contra de la empresa brasileña Odebrecht que, desde 2010, amenaza con destruir el sistema hídrico de la cuenca La Antigua; y la visibilización de los problemas agrarios debido a la corrupción y a la mala administración estatal y municipal (Baca-Feldman, 2014); formas de cooperación autónomas frente a intereses ajenos a la clase, la incorporación institucional y la racionalidad económica.

Cuando la constructora brasileña intentó represar el río de la región, Radio Teocelo fue un medio que favoreció la coordinación entre localidades, grupos organizados y prestadores de servicios que vieron amenazados sus intereses y sus territorios. La construcción de una identidad colectiva, basada en el sentido de comunidad, posibilitó la definición de expectativas y el cálculo de costos/beneficios de la acción colectiva, que respondió mediante estructuras organizacionales de cooperación ante una perturbación en el entorno social. Riveros afirma que este ha sido:

... un muy buen ejemplo de cómo un medio comunitario puede incidir, precisamente, en una estrategia de autodefensa: defender el río, defender la economía local y regional, evitar el destrozo de la zona, de alterar una identidad, de imponer una forma de consumir y de organizar a la gente.

Lejos de una determinación tecnológica, Riveros concuerda, las conquistas sociales no dependen de forma exclusiva de los medios. La transformación social obedece a los sentidos 


\section{Balajú}

de producción y codificación de mensajes, asentados sobre la identidad colectiva (Atton, 2002). No son los medios comunitarios quienes resuelven los problemas: son las personas que los apropian, quienes se informan y se organizan a través de estos. Por su parte, Baca-Feldman (2014) ha apuntado que las comunidades, a través de su radio, no solo se enfrentan a la clase política; las condiciones del estado la han llevado a enfrentar a grupos delictivos que buscan romper con las dinámicas comunitarias de acción colectiva. El ejercicio de los medios comunitarios también puede implicar consecuencias en este sentido, un aspecto a destacar en cuanto a la misión de sus comunicadores y sus comunidades.

Por todo lo anterior, a través de la extensión del ejercicio cotidiano y de los contenidos de Radio Teocelo, esta se reafirma como una tecnología apropiada por la comunidad para la constitución de una esfera pública alternativa, para la deliberación, la representación de la identidad, y la coordinación de acciones colectivas orientadas por la intencionalidad de sus actores y enfocadas en intereses, objetivos y problemas comunes. De igual forma, se han evidenciado las posibilidades de incidencia en el amplio espacio político, de la opinión pública producida por la sociedad civil, mediante prácticas mediáticas a través de Radio Teocelo.

El reconocimiento de Radio Teocelo como una esfera pública alternativa no representa un fin; por el contrario, se establece como una plataforma de visibilización, reconfigurada por múltiples comunidades y movimientos sociales, ante diversos contextos y situaciones: la defensa del medio ambiente, la lucha contra la corrupción y la mala administración de los recursos naturales, todo orientado hacia la preservación de la identidad colectiva. Las conquistas logradas han sido el resultado de acciones colectivas de actores y comunidades que se han movilizado y han apropiado la radio para generar y visibilizar su opinión respecto a asuntos de interés. Radio Teocelo ha abonado al sentimiento de comunidad mediante procesos de reconocimiento de una identidad colectiva que revitaliza lazos de solidaridad y a través de redes de interacción orientadas hacia bienes que escapan a la lógica institucional y mercantil. La comprensión de estas acciones colectivas va más allá de una lógica materialista y acumulativa, por lo cual su análisis se adecua al concepto de NMS propuesto por Melucci (1999).

\section{Conclusión}

En principio, se reconoce la condición performativa de la comunidad como una estructura simbólica de relaciones sociales, inserta en un contexto dominante, un espacio de resistencia y 


\section{Balajú}

mediación donde se desarrolla la vida social, basado en procesos de reconocimiento y de diferenciación que producen una identidad común, desde la cual es posible generar acciones colectivas hacia objetivos e intereses compartidos. De acuerdo con su carácter subjetivo, se trascienden demarcaciones geográfico-espaciales, sin comprometer los sentidos de identidad y de solidaridad que hacen posibles acciones de apropiación y resignificación de espacios y tecnologías.

Se identifican las experiencias de apropiación y de reconfiguración de tecnologías de comunicación como recursos en el campo de acción colectiva para la movilización hacia objetivos e intereses compartidos. El punto de partida de esta perspectiva es la acción social de una comunidad, delimitada por un horizonte simbólico desde el cual calcular los costos/beneficios y reconocer las oportunidades de acción de los individuos que buscan modificar su contexto, de forma autónoma frente a la intervención estatal.

En ese sentido, en tanto su acceso y su participación no estén condicionados por mecanismos de exclusión ni por relaciones de dominación o subordinación, la radio comunitaria puede constituir un espacio público alternativo, asentado sobre redes de interacción y de comunicación sustentadas en una identidad comunitaria. Un espacio autónomo a la esfera pública central, producido por acciones colectivas, con aforo para la deliberación y la organización, para publicitar un producto de representatividad de objetivos, intereses e identidades comunes.

De esta forma, se identifica a Radio Teocelo como una tecnología apropiada por la acción colectiva, en respuesta a desequilibrios sistémicos delimitados por el horizonte simbólico de experiencias y significados comunes. Como apropiación, Radio Teocelo derivó de una identidad colectiva que evidenció la incapacidad y el desinterés institucional y de los medios comerciales por satisfacer las necesidades sociales e informativas. Así, desde sus inicios la emisora ha mantenido una dinámica de integración que ha hecho posible su apropiación dentro del repertorio de acciones de múltiples comunidades y movimientos sociales, y que ha favorecido diversas formas de organización, movilización y acción no institucionales.

Radio Teocelo constituye un espacio público -alternativo o subalterno- de colaboración y de organización para el refuerzo de identidades colectivas, mediante la publicidad de expresiones culturales y políticas de múltiples comunidades, más allá del municipio de Teocelo, identidades intersubjetivas que han producido experiencias inéditas de 


\section{Balajú}

comunitarismo mediante lazos de correspondencia y de solidaridad, incluso a través de geografías diferenciadas. La operación de la emisora está sustentada en formas anteriores de comunicación e interacción sobre redes de identidad, correspondencia y solidaridad afianzadas en la vida cotidiana; su ejercicio y subsidio dependen de la participación comunitaria.

Como esfera pública alternativa para la deliberación y la acción social, Radio Teocelo ha sido una plataforma autónoma de diálogo y conflicto con otras esferas centrales e institucionales. Dentro del campo de posibilidades y límites de las acciones colectivas, sus capacidades se han extendido hacia procesos sociales de mayor amplitud: la defensa del territorio, la visibilización de problemas agrarios, la mala administración estatal y municipal e intereses ajenos a la racionalidad institucional y económica. Sin embargo, las conquistas no han sido determinadas por el medio; derivan de acciones de organización y de coordinación que han apropiado y resignificado las tecnologías dentro de su repertorio de acciones. Así, diversos actores sociales y comunidades han representado sus reivindicaciones a través de Radio Teocelo y han coordinado acciones colectivas hacia objetivos comunes, producto de la mediación de aspectos identitarios.

El análisis de los medios como espacios de acción social facilita la comprensión de las prácticas mediáticas que han favorecido la representación de ideales políticos, así como el diálogo entre múltiples actores que en esas esferas se celebra. Dichas acciones amplían las capacidades para representar los procesos de resignificación de mensajes y las formas de relación política a través de tecnologías de comunicación.

Por otra parte, el análisis de medios a la luz de los NMS permite incorporar un enfoque ecologista que tome en cuenta un ecosistema mediático en el cual los actores extienden prácticas comunicativas, se organizan y despliegan un variado repertorio de acciones simbólicas a través de múltiples dispositivos de comunicación, de manera simultánea. Asimismo, permite constatar la evolución de prácticas que han transitado desde formas interpersonales de comunicación e interacción hacia distintos dispositivos y sistemas de significación que interactúan entre sí. A partir de formas de relación generadas en un colectivo social, la comunidad de Teocelo ha apropiado diversas tecnologías dentro de su repertorio de acciones, con el fin de representar sus objetivos, identidades e intereses: Radio Teocelo, el periódico Altavoz y Teocelo Te Ve. Estos medios han interactuado entre sí y han compartido contenidos; incluso, a pesar de la extinción de Altavoz, sus contenidos han transitado a otros sistemas. Aunque la atención se centró en la emisora de radio, es fundamental tomar en cuenta 


\section{Balajú}

las posibilidades de apropiación sobre diversos dispositivos mediáticos, derivadas de sistemas previos de relación mediante los cuales las comunidades dialogan y se organizan.

Por último, Radio Teocelo ha sido una tecnología apropiada por la comunidad, dentro de un amplio ecosistema de relaciones y de medios. De igual forma, su esquema de organización y su sistema de producción y consumo también son, con constancia, reconfigurados y mediados de acuerdo con los actores sociales y movimientos que la intervienen en distintos contextos y situaciones. No obstante, el logro de los objetivos y la resolución de problemáticas comunes no son resultado exclusivo del consumo y la codificación preferente de sus contenidos. Por el contrario, derivan de movilizaciones sociales y de acciones colectivas heterogéneas y dinámicas, enfocadas en intereses, objetivos y problemas comunes: las personas que por una voluntad emancipadora apropian y reconfiguran los dispositivos que se encuentran en su campo de posibilidades, para enfrentar las tensiones que disturban el equilibrio estructural de su entorno (Melucci, 1999).

\section{Referencias}

ALMEIDA, E. y M. Sánchez (2014). Comunidad: Interacción, conflicto y utopía. Universidad Iberoamericana.

Altavoz (4 de septiembre de 2015). "50 años al aire... ¿Continuamos?" Altavoz en línea. http://periodicoaltavoz.blogspot.com/2015/09/50-anos-al-aire- continuamos.html ARATO, A. y J. Cohen (2001). Sociedad civil y teoría política. Fondo de Cultura Económica. ARIAS-GODÍNEZ, B. (1990). La XEYT, radio cultural campesina. Una experiencia de radio participativa. Asociación Latinoamericana de Educación Radiofónica.

Asociación Mundial de Radios Comunitarias-México (AMARC-MX) (2008). Radios comunitarias y contexto de conflicto en México. AMARC-MX. http://library.fes.de/pdffiles/bueros/mexiko/06445.pdf

ATTON, C. (2002). Alternative media. Sage.

BACA-FELDMAN, C. (2014). "Pensando ‘otra comunicación’. Radio comunitaria en México, un abordaje desde la teoría crítica", en P. Gentili (ed.), Medios alternativos y movimientos sociales. Consejo Latinoamericano de Ciencias Sociales, 89-110.

BERRÍO, A. (2006). "La perspectiva de los nuevos movimientos sociales en las obras de Sydney Tarrow, Alain Touraine y Alberto Melucci”, Estudios Políticos 29, 218-236. https://www.redalyc.org/articulo.oa?id=16429057009 
CAlleJA, A. y B. Solís (2005). Con permiso: la radio comunitaria en México. Fundación Friedrich Ebert-México.

CASSIGOLI, R. (2016). “Antropología de las prácticas cotidianas: Michel de Certeau”, Chungara. 84 (4), 679-690. http://dx.doi.org/10.4067/S0717-73562016005000033

CAUSSE, C. (2009). "El concepto de comunidad desde el punto de vista socio-históricocultural y lingüístico", Ciencia en su PC 3, 12-21. https://n9.cl/rw0k

CERTEAU, M. de (2000). La invención de lo cotidiano I. Artes de hacer. Universidad Iberoamérica.

CERTEAU, M. de (2000). La invención de lo cotidiano 2. Habitar, cocinar. Universidad Iberoamérica.

CONAPRED (2010). Las radios comunitarias como mecanismo en contra de la discriminación. https://www.conapred.org.mx/documentos_cedoc/E15-2009.pdf

DIANI, M. (1992). “The Concept of Social Movement”, The Sociological Review, 40 (1), 125. https://doi.org/10.1111/j.1467-954X.1992.tb02943.x

DOWNING, H. (2000). Radical Media: Rebellious communication and social movements. Sage.

DUARTE, C. (2017). Trabajo social comunitario: perspectivas teóricas, metodológicas, éticas y políticas. Tesis doctoral, Universidad Complutense de Madrid.

FONTANA, A. y J. Frey (2012) "La entrevista. De una proposición neutral al compromiso político”, en N. Denzin y Y. Lincoln (coords.), Manual de investigación cualitativa. Volumen I, Gedisa, 141-202.

FRASER, N. (1999). "Repensando la esfera pública: una contribución a la crítica de la democracia actualmente existente", Debate, 46, 136-174. https://n9.cl/0v0wu

GARCÍA CANCLINI, N. (2006). "El consumo cultural: una propuesta teórica", en G. Sunkel (comp.), El consumo cultural en América Latina. Construcción teórica y líneas de investigación. Convenio Andrés Bello, 26-49.

GASPARELLO, G. (2011). "Donde crece la flor de la palabra. Reflexiones sobre la radio comunitaria indígena en los estados de Guerrero y Oaxaca, México”, Nómadas, 29 (1), 347-364. http://dx.doi.org/10.5209/rev_NOMA.2011.v29.n1.26816

GROSSI, G. (2007). La opinión pública: Teoría del campo demoscópico. Centro Investigación Sociológica. 


\section{Balajú}

GUMUCIO, A. (2004). "El cuarto mosquetero: la comunicación para el cambio social", Investigación \& Desarrollo. 12 (1), 2-23. https://n9.cl/xm9d

GUO, L. (2015). "Exploring the Link between Community Radio and the Community: A Study of Audience Participation in Alternative Media Practices", Communication, Culture and Critique, 10 (1), 112-130. https://doi.org/10.1111/cccr.12141

HABERMAS, J. (1981). Historia y crítica de la opinión pública. Gustavo Gil.

HABERMAS, J. (1998). Facticidad y validez. Sobre el derecho y el Estado democrático de derecho en términos de teoría del discurso. Trotta.

HERNÁNDEZ, G. (2013). La digitalización de la radio en México. Tesis de licenciatura, Universidad Nacional Autónoma de México. https://n9.cl/1ib6r

HERNÁNDEZ, R., C. Fernández y P. Baptista (2014). Metodología de la investigación. McGraw-Hill.

KOOPMANS, R. (2004). "Movements and Media: Selection Processes and Evolutionary Dynamics in the Public Sphere", Theory and Society, 33 (3/4), 367-391. https://doi.org/10.1023/B:RYSO.0000038603.34963.de

KORSBAEK, L. (2009). "El comunalismo: cambio de paradigma en la antropología mexicana a raíz de la globalización", Argumentos, 22 (59), 101-123. https://n9.cl/cajqp

KROHLING, C. (2012). "Aproximaciones entre la comunicación popular y comunitaria y la prensa alternativa en Brasil en la era del ciberespacio", en M. Martínez y F. Sierra (coords.), Comunicación y desarrollo: prácticas comunicativas y empoderamiento local. Gedisa, 112-125.

LAMAS, E. (2003). Gestión integral de la radio comunitaria. Fundación Friedrich Ebert.

LARAÑA, E. (1999). La construcción de los movimientos sociales. Alianza.

LIEVROUW. L. (2011). Alternative and Activist New Media. Polity Press.

LÓPEZ, J. (1995). “QQué hace comunitaria a una radio comunitaria?” Chasqui. Revista Latinoamericana de Comunicación, (52), 51-54. https://n9.cl/4wa4w

MARTÍN-BARBERO, J. (1987). De los medios a las mediaciones. Comunicación, cultura y hegemonía. Gustavo Gili.

MASIP, F. (2011). “Comunidades aporéticas”, Tramas. Subjetividad y Procesos Sociales, 34, 47-73. https://biblat.unam.mx/hevila/TramasMexicoDF/2011/no\%2034/2.pdf 


\section{Balajú}

MATTONI, A. (2017). "A Situated Understanding of Digital Technologies in Social Movements. Media Ecology and Media Practice Approaches", Social Movement Studies, 16 (4), 494-505. https://doi.org/10.1080/14742837.2017.1311250

MATTONI, A. y E. Treré (2014). "Media Practices, Mediation Processes, and Mediatization in the Study of Social Movements", Communication Theory, 24 (3), 252-271. https://doi.org/10.1111/comt.12038

MATTONI, A. y E. Treré (2016). "Media Ecologies and Protest Movements: Main Perspectives and Key Lessons", Information, Communication and Society, 19 (2), 290306. https://doi.org/10.1111/comt.12038

MEJÍA, F. (1989). La industria de la radio y la televisión y la política del estado mexicano: 1920-1960. Fundación Manuel Buendía.

MELUCCI, A. (1999). Acción colectiva, vida cotidiana y democracia. El Colegio de México.

MHAGAMA, P. (2015). "Radio Listening Clubs in Malawi as Alternative Public Spheres", The Radio Journal. International Studies in Broadcast \& Audio Media, 13 (1), 105120. https://dx.doi.org/10.1386/rjao.13.1-2.105_1

MILAN, S. (2008). "What Makes You Happy? Insights into Feelings and Muses of Community Radio Practitioners", Westminster Papers in Communication and Culture, 5 (1), 25-43. http://doi.org/10.16997/wpcc.49

MILAN, S. (2009). "Four Steps to Community Media as a Development Tool", Development in Practice, 19 (4/5), 598-609. https://dx.doi.org/10.1080/09614520902866421

MILAN, S. (2013). Social Movements and Their Technologies. Wiring Social Change. Palgrave Macmillan.

MONJE, C. (2011). Metodología de la investigación cuantitativa y cualitativa. Guía didáctica. Universidad Surcolombiana.

NIGHTINGALE, V. (2011). The Handbook of Media Audiences. Blackwell Publishing.

OLMEDO, R. (2016). "Radio Teocelo. Un caso de apropiación social de los medios de comunicación”, en R. Ibarra (presidencia), Trascender el neoliberalismo y salvar a la humanidad. Ponencia presentada en el IV Congreso Latinoamericano de Ciencias Sociales. Universidad Autónoma de Zacatecas.

PARRA, N. (2017). Aproximaciones al concepto de comunidad para el Trabajo Social. https:// doi: 10.13140/RG.2.2.27154.35522 


\section{Balajú}

PEPPINO, A. (1990). La Radio permisionada en México: el caso de Hidalgo. Fundación Friedrich Ebert.

RABOTNIKOF, N. (2011). En busca de un lugar común. Universidad Nacional Autónoma de México.

RAMOS, V. (2007). "La radio comunitaria frente a los grupos de poder", Razón y Palabra, 59. https://www.redalyc.org/articulo.oa?id=1995/199520703006

REVILLA, B. (1996). "El concepto de movimiento social: acción, identidad y sentido", Última Década, 5, 1-18. https://n9.cl/n589u

RODRÍGUEZ, C. (2011). “Trayectoria de un recorrido: comunicación y cambio social en América Latina", en J. Pereira y A. Cadavid (eds.), Comunicación, desarrollo y cambio social. Pontifica Universidad Javeriana, 37-56.

ROJAS, C. (2011). "Comunidad y movimientos sociales", Universitas 15, 197-216. https://doi.org/10.17163/uni.n15.2011.07

ROMO, C. (1991). Ondas, canales y mensajes: un perfil de la radio en México. Instituto Tecnológico y de Estudios Superiores de Occidente.

SAMPEDRO, V. (2000). Opinión pública y democracia deliberativa. Medios, sondeos y urnas. Ediciones Istmo.

SCOLARI, C. (2012). "Media Ecology: Exploring the Metaphor to Expand the Theory", Communication Theory, 22 (2), 204-225. https://doi.org/10.1111/j.14682885.2012.01404.x

SEID, G. (2016). "Procedimientos para el análisis cualitativo de entrevistas. Una propuesta didáctica", V Encuentro Latinoamericano de Metodología de las Ciencias Sociales (ELMCS), Mendoza, Argentina. https://n9.cl/pi913

SERMEÑO, A. (2013). "Razón y espacio público en la democracia deliberativa. Una perspectiva habermasiana", Andamios, 10 (23), 205-231. https://n9.cl/9buum

SOCARRÁS, Elena (2004). "Participación, cultura y comunidad", en Linares Fleites, Cecilia, Pedro Emilio Moras Puig y Bisel Rivero Baxter (comps.), La participación. Diálogo y debate en el contexto cubano. La Habana: Centro de Investigación y Desarrollo de la Cultura Cubana Juan Marinello, 173-180.

SOSA, J. (2011). El movimiento radiofónico de comunicación popular en América Latina: el caso de Radio Teocelo, Veracruz. Universidad Nacional Autónoma de México. 
TRERÉ, E. (2012). “Social Movements as Information Ecologies: Exploring the Coevolution of Multiple Internet Technologies for Activism", International Journal of Communication, 6, 2359-2377. https://ijoc.org/index.php/ijoc/article/view/1681

VIOLICH, F. y J. Astica (1971). Desarrollo de la comunidad y el proceso de planificación urbana en la América Latina. University of California. 